\title{
The simulation of fabric development during plastic deformation and its application to quart- zite: the influence of deformation history
}

\author{
G. S. LISTER \\ Department of Structural Geology, Institute of Earth Sciences, University of Utrecht, Utrecht, The Netherlands \\ and \\ B. E. HовBS
}

Department of Earth Sciences, Monash University, Clayton, Melbourne, Victoria, 3168, Australia

(Received 5 July 1979; accepted in revised form 24 January 1980)

\begin{abstract}
The effect of deformation history on the development of crystallographic preferred orientation in quartzities has been simulated using a computer program based on the Taylor-Bishop-Hill analysis. Model quartzities with different combinations of glide systems have been subjected to various coaxial and non-coaxial deformation histories. It is possible to obtain information from the fabrics that develop during simple histories; for example, the location of the axis of extension is generally associated with a pole free area on a c-axis plot, and progressive axial shortening, plane strain and axial shortening produce characteristic fabrics. In progressive simple shear the fabric skeleton becomes asymmetric relative to the sense of shear and $a$-axes preferentially align in the flow plane parallel to the flow direction. However, this example illustrates that the fabric orientation and characteristics are controlled by the kinematic framework and bear only an indirect relationship to the finite strain accumulated to that point in the history.

The imprint of the closing stages of deformation limits to some degree the use of crystallographic fabrics as a tool for structural geologists, but in favourable circumstances data can be obtained concerning characteristics of the deformation history, on the scale of the hand-specimen, for the last part of this history.
\end{abstract}

\section{INTRODUCTION}

UNDER conditions of plastic deformation of polycrystalline material where intracrystalline slip plays an important role, the crystal axes may tend to rotate and a pattern of preferred orientation can develop. The lattice rotations develop because the crystal structure of a crystalline material deforming through the conservative migration of dislocations remains more or less undistorted, though the grains themselves undergo gross changes in shape. Thus, the crystal axes act as an internal reference frame during deformation. Provided that deformation within a specified crystalline volume is statistically homogeneous, the deformation induced by glide systems can be described by inter-penetrating simple shears. This means that, from a kinematic viewpoint, the crystalline material develops a vorticity with respect to the crystal axes (see Lister et al. 1978). However, the geometrical constraints of deformation may impose a different vorticity on the material, this vorticity being expressed with respect to some set of axes external to the crystal. In consequence the crystal axes spin relative to the external axes so that the externally imposed vorticity is maintained.

These relations can be expressed by means of the equation:

$$
\dot{\mathbf{\Omega}}_{C}^{E}=\dot{\mathbf{\Omega}}_{M}^{E}-\dot{\mathbf{\Omega}}_{M}^{C},
$$

which simply states that the vorticity tensor $\dot{\Omega}_{C}^{E}$, describing the rate of rotation of the crystal axes $(C)$ with respect to an external frame $(E)$, is the difference between the vorticity $\dot{\Omega}_{M}^{E}$ of the material expressed relative to this external frame and the vorticity $\dot{\Omega}_{M}^{C}$ of the material expressed relative to the crystal axes.

Lattice reorientation as a result of dislocation glide may be responsible for many of the crystallographic fabrics observed in strongly deformed quartzites and it is important to attempt to understand the effects of deformation by dislocation glide on the development of patterns of preferred orientation. Recrystallization and grain growth are important in nature, but in many instances it appears that these processes exert 'only a modifying influence on pre-existing, or co-developing deformation fabrics (see Bell \& Etheridge 1976, Lister \& Price 1978).

In applying a computer program based on the Taylor-Bishop-Hill analysis (see Lister et al. 1978) the following factors have been found to affect the development of deformation fabrics: (i) the initial orientation distribution; (ii) the deformation history by which the final deformed state was achieved; and (iii) the dislocation glide systems, and combinations of glide systems, that operate to allow deformation. This paper is concerned with the effect of deformation history according to the Taylor-Bishop-Hill analysis. Previous papers, Lister et al. (1978) and Lister \& Paterson (1979), have dealt with the general Taylor-Bishop-Hill theory and with fabric transitions.

Experiments with quartz single crystals have indicated many possible dislocation glide systems, including in particular, systems with $<c+a>$ Burgers vector (Baeta \& Ashbee 1969 a, b, 1970, Morrison-Smith et al. 1976) and even the $\langle c+2 a\rangle$ vector (Twiss 1976). The sys- 
Table 1. Dislocation glide systems used in the fabric simulations. From left to right for each glide system are shown: the Miller index of the glide plane: the Burgers vector, referring to the $a_{1}, a_{2}$ and $c$ axes: hexagonal Miller indicies; and the algebraic representation of the slip vector.

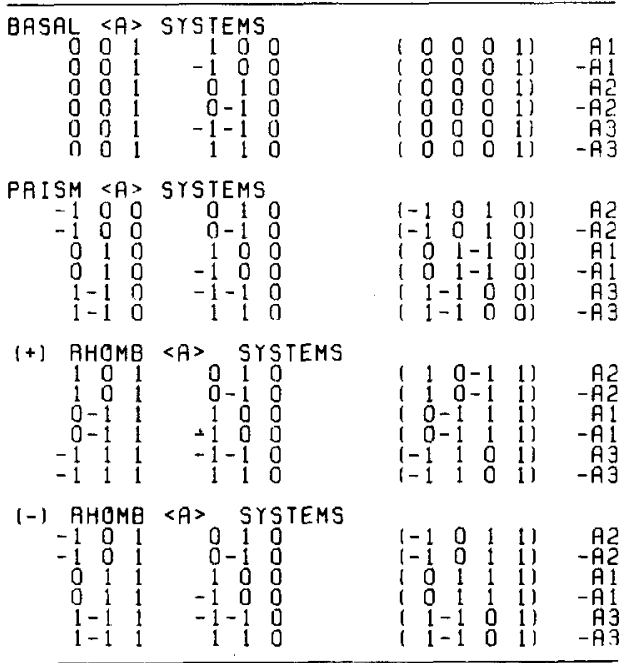

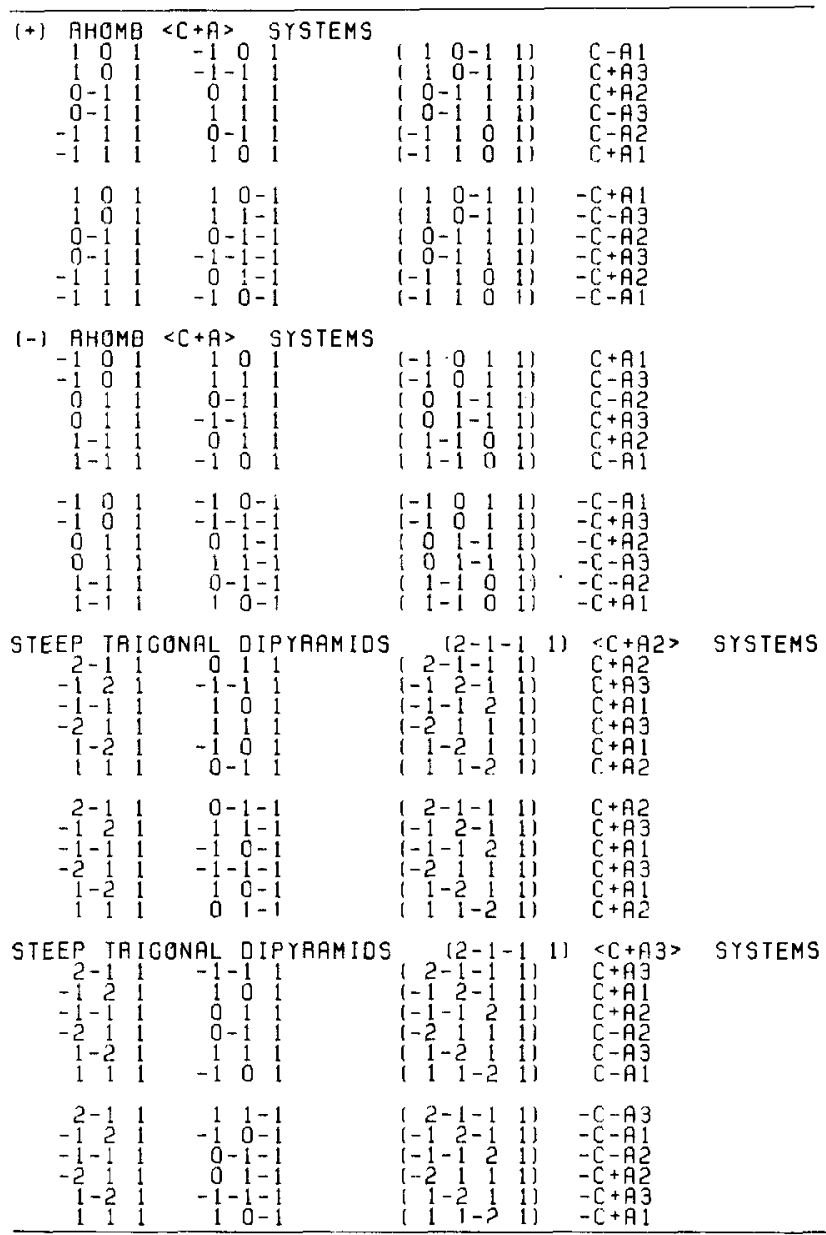

ously and which cannot. An important point to note is that it is the relative and not the absolute values of the critical yield stresses that govern the development of fabric.

The analysis assumes each grain is rigid until shear stresses resolved on one or more slip planes in the direction of slip reach critical values. Then the crystal behaves in a perfectly plastic manner. Rigid-plastic behaviour implies the existence in stress space of a yield surface that bounds all of those stress states for which the crystal is rigid. Stress states on the yield surface are the only possible ones allowing plastic deformation. Thus the stress within a grain cannot be arbitrarily specified, nor need it bear a straightforward relationship to the imposed strain state; the possible stress states within a grain are pre-determined by the choice of critical resolved shear stresses.

The yield surface is formed by the intersections of 'planar' segments (hyperplanes in stress space), each plane corresponding to the yield constraint for a particular dislocation glide system. Each constraint is defined by a linear inequality of the form:

$$
\sigma_{i j} n_{i}^{\alpha} l_{j}^{\beta} \leqslant \tau_{\text {critical }}^{\alpha \beta}
$$

which states that the stress within the grain, $\sigma_{i j}$, resolved on the glide plane, $\alpha$ (whose normal is $n^{\alpha}$ ) and in the $\beta$ slip direction, $\boldsymbol{I}^{\boldsymbol{\beta}}$, must be less than or equal to the critical a specific choice of systems, the relative yield stresses determine which combinations can operate simultane-
Once the set of dislocation glide systems has been specified for a fabric simulation, one must specify critical mechanisms are able to operate. This choice determines the primary characteristics of any pattern of preferred orientation that results from a computer simulation. Distinctive lattice rotations are produced when particular combinations of glide systems operate, and given

tems used in this study are listed in Table 1. For
geometric reasons the employment of systems with Burgers vector such as $\langle c+a\rangle$ is essential for the application of the Taylor-Bishop-Hill analysis. This arises lished by conservative glide of dislocations alone unless such systems operate (see Paterson 1969). Since the evidence for the existence of these systems is not as clear as tems, the fact that we are obliged to use the $\langle c+a\rangle$ vector is a point to note when considering the implica-

\section{DETAILS OF MODELS ADOPTED}

\section{The yield surface configuration}


resolved shear stress for that system, $\tau_{\text {critical. If the }}^{\alpha \beta}$ resolved shear stress is less than $\tau_{\text {critical }}$ then the crystal is rigid; if the two are equal the crystal deforms in a plastic manner. Thus, the distance that any particular constraint hyperplane lies from the origin in stress space is equal to the critical resolved shear stress (CRSS) value for the corresponding slip system. The yield surface is therefore defined by the intersection of a set of linear inequalities in stress space and consists of segments of those constraint hyperplanes closest to the origin, i.e. those with the lowest CRSS values. If the CRSS value for one system is relatively high in comparison with the values for other systems, and the system is not geometrically indispensable, it may be prevented from operating because the corresponding yield constraint is excluded from the yield surface.

It is evident from these arguments that the list of CRSS values determines which glide systems operate and further, since stress states at the vertices of the yield surface activate two or more glide systems simultaneously, the CRSS values also determine which combinations of glide systems can operate together. Hence, the vertices of the yield surface are the single most important factor in determining how crystallographic fabrics develop, according to the Taylor-Bishop-Hill theory.

For a particular imposed strain increment the analysis determines which stress state will apply during deformation. For a general strain increment this stress must lie at a vertex of the yield surface determined by the intersection of at least five constraint hyperplanes. The vertex determines which glide systems operate and the relative activities of the operating systems are then decided by their geometical relations with the imposed strain vector, in six-dimensional strain-increment space. The CRSS values then play no further role in the calculation. Note that in the case of five glide systems only (assuming these are linearly independent) the shape of the vertices does not change at all when the relative CRSS values alter. Mechanism activity is in this case solely determined by geometry.

At first sight it may appear that the yield surface has as many different shapes as there are different lists of CRSS values and hence choosing one or another CRSS list for a simulation is somewhat arbitrary. However, the CRSS values can vary over a considerable range and not alter the topological configuration of the vertices of the yield surface. As long as the configuration of the vertices does not change, different lists of CRSS values result in the same fabric for the same imposed deformation history. However, if the CRSS values are changed to a sufficient degree, the configuration of the yield surface will be altered to an extent that different vertices appear.
Different combinations of mechanisms can then be activated to allow the same deformation and in general different patterns of preferred orientation will result. In other words, different yield surface vertex configurations lead to different fabrics for the same deformation histories; different lists of CRSS values lead to different yield surfaces but not necessarily different vertex configurations.

\section{Choice and results for three model quartzites}

Attempting to determine the range of fabric variation predicted by the Taylor-Bishop-Hill analysis poses three separate problems: (i) writing down the relevant list of dislocation glide systems; (ii) determining all the possible yield surface configurations; and (iii) characterizing the most important of these configurations for the effects of deformation history. Our analysis has been applied in the classical way, i.e. assuming strictly homogeneous strain, and leaving CRSS values constant throughout a simulation. It would be possible to ascertain the effects of alternative and perhaps more realistic conditions, for example: (i) inhomogeneous strain; and (ii) dynamically determined CRSS values; considering the interaction between glide systems, with workhardening or softening of active systems, and latent hardening or softening of inactive ones. So far we have not attempted these non-classical applications in a systematic fashion.

If restrictive assumptions are not to be made, the list of glide systems to consider may have up to 22 degrees of freedom in the assignment of CRSS values (see Lister \& Paterson 1979 for a detailed discussion). To discover all the possible yield surface configurations is a task beyond our computing resourses so, as yet the total variation is not documented, and is only partially understood.

Three hypothetical model quartzites were defined for this study by specifying three sets of CRSS values for the systems listed in Table 1 . These values are shown in Table 2. Some effort has been directed toward choosing models that produce fabrics similar to those found in nature and in experiments.

(i) Model quartzite A. Model A is representative of a family of yield surface configurations that gives rise to a point maximum of $c$-axes parallel to $Z$ for progressive axially symmetric shortening (using the convention $X$, $Y, Z$ for the principal axes of finite strain, where $X$ is the axis of extension, $Y$ the axis of intermediate strain, and $Z$ the axis of shortening). Such fabrics are common in nature and were also obtained in the experiments of Tullis et al. (1973) for lower temperature deformations

Table 2. CRSS values used for each of the three model quartzites.

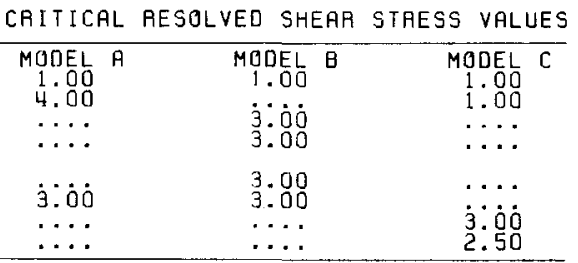


and faster strain rates. Model A produces double wedge patterns in progressive plane strain (cf. Tullis 1977) and cleft girdles in axially symmetric extension (cf. Weiss 1954). The essential characteristic of this model is that basal $<\boldsymbol{a}>$ systems are relatively soft, and the CRSS ratio for the positive and negative rhombs is high. In fact, in this model positive rhombs were not allowed to operate at all (see Lister $e$ al. 1978). This has the effect of producing a preferred orientation of positive forms parallel to the shortening axis (Fig. 1 shows the inverse pole figures for progressive axial shortening, plane strain and axial extension). A similar tendency was noted by Tullis et al. (1973) but it is not possible to compare these theoretical pole figures with the experimental ones until some way is found of accounting for the presence of different bands of quartz, and for the effects of Dauphine twinning (see Tullis \& Tullis 1972). The effect of prism $<a>$ glide is to produce concentrations of $c$ axes at high angles to the axis of shortening. If the prism system is made softer than in model $A$, stronger concentrations of $c$-axes at high angles to $Z$ result. If the CRSS value on the prisms is increased from 4 to 6.5 prism activity ceases, and there is then no concentration of $\boldsymbol{c}$-axes at high angles to $Z$ at all. However, Tullis $e t a l$.

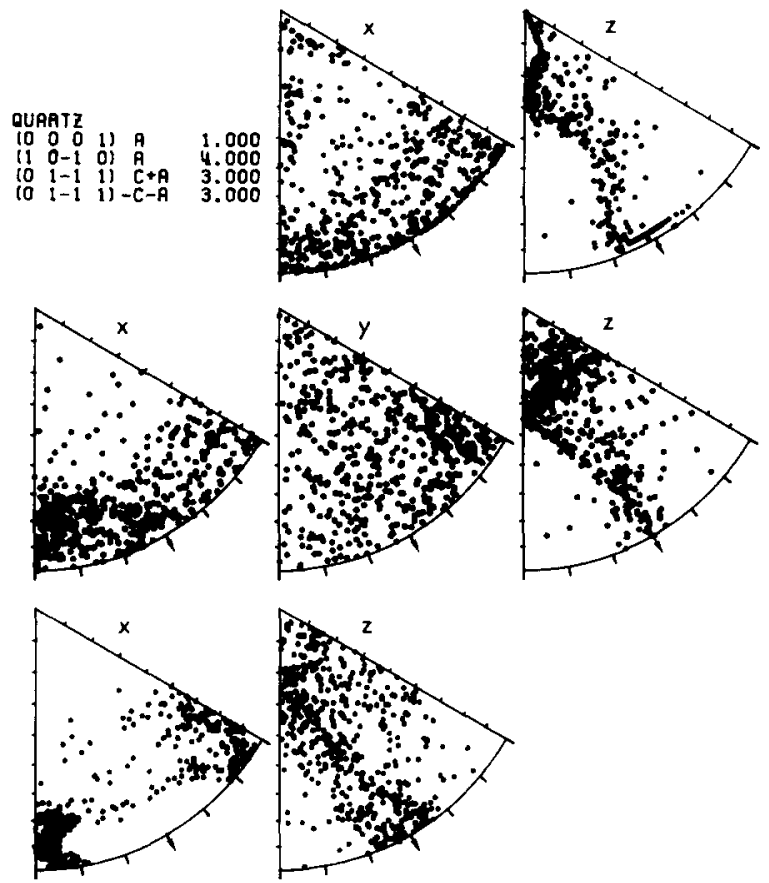

Fig. 1. Model quartzite A subjected to progressive axially symmetric shortening (top row), plane strain (middle row) and axially symmetric extension (bottom row). Each simulation involves 14 increments and the deformation gradients used are set out in Table 3 . In plane strain this deformation involves $50 \%$ shortening in total. Inverse pole figures show the distribution of $X$, the axis of extension, $Y$, the axis of intermediate strain, and $Z$, the axis of shortening after 14 increments for the three deformation histories above, with respect to crystallographic axes. The $c$-axis is vertical, the a-axis marked, and positive forms occupy the left-half of the segment of the upper hemisphere equal area projection used. CRSS values are listed.

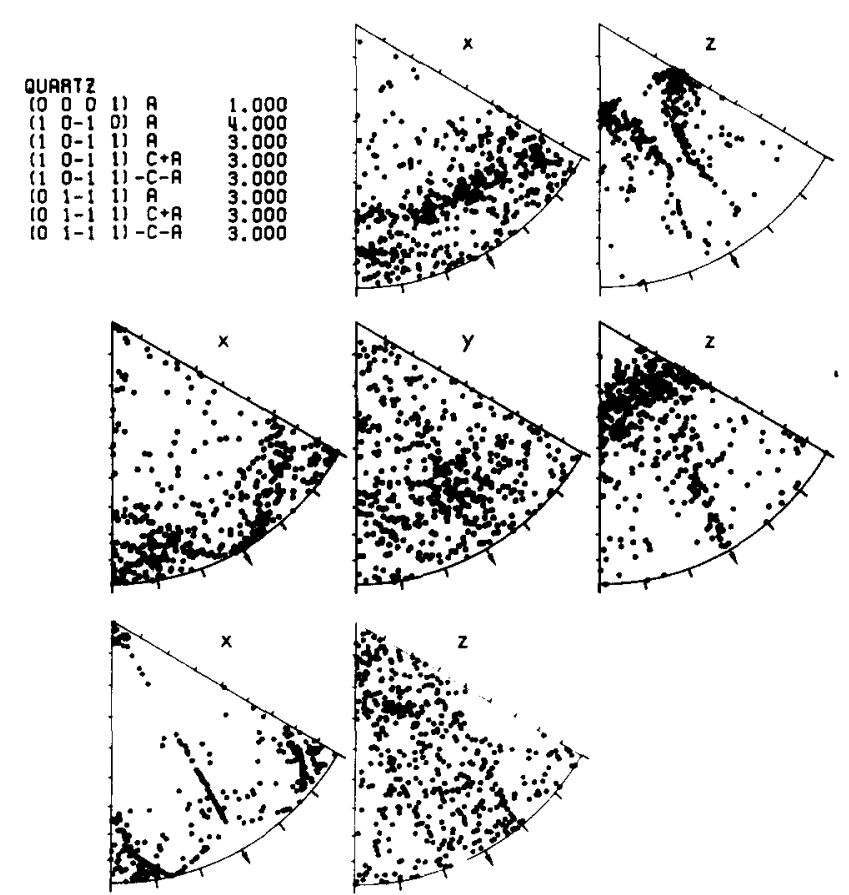

Fig. 2. Model quartzite B subjected to various progressive deformations. Details are the same as in Fig. 1.

(1973) show a slight concentration of c-axes at high angles to $Z$, so the CRSS ratio on the basal $\langle a\rangle$ and prism $<a>$ systems was set to mimic that tendency.

(ii) Model quartzite B. Model B is representative of a family of yield surface configurations that gives rise to a $25^{\circ}$ small-circle girdle of $c$-axes around $Z$, for progressive axially symmetric shortening (inverse pole figures are shown in Fig. 2). For progressive plane strain a double wedge pattern develops, just as for model A, but the small-circle girdle about $Z$ develops with different characteristics, including: (a) a pole free area at $Z$; and (b) maxima between $Z$ and $Y$ instead of between $Z$ and $X$. Model B produces a fabric that compares with the type I crossed-girdle or double maximum of c-axes commonly found in quartz tectonites (cf. Christie 1963, Hara et al. 1973, Riekels \& Baker 1977). The opening angle of the girdles and the angle between the maxima and $Z$ are however, lower than for most natural fabrics. The essential characteristic of model $B$ is that basal $<a>$ systems are relatively soft, and the positive and negative rhomb $<c+a>$ systems are equally hard. Although prism $<a\rangle$ systems are still included with the same CRSS value as in model $A$, their activity is now negligible. Model B slip systems are compatible with the quartz being in the $\beta$-quartz field.

(iii) Model quartzite C. Model C is representative of a family of yield surface configurations that gives rise to a $40-50^{\circ}$ small-circle girdle of $c$-axes around $Z$ for

Table 3. Deformation gradients applicable to increments used in simulations.

\begin{tabular}{llll}
\hline TYPE OF STRAIN HISTORY & \multicolumn{3}{c}{ PAINCIPAL STRETCHES } \\
AXIALLY SYMMETRIC EXTENSION & 1.050 & 0.975 & 0.975 \\
CONSTRICTIYESTRAIN & 1.050 & 0.988 & 0.963 \\
PLANESTRAIN & 1.050 & 1.000 & 0.950 \\
FLATENING STRAIN & 1.037 & 1.013 & 0.950 \\
AXIALLYSYMMETAIC SHORTENING & 1.025 & 1.025 & 0.950 \\
\hline
\end{tabular}




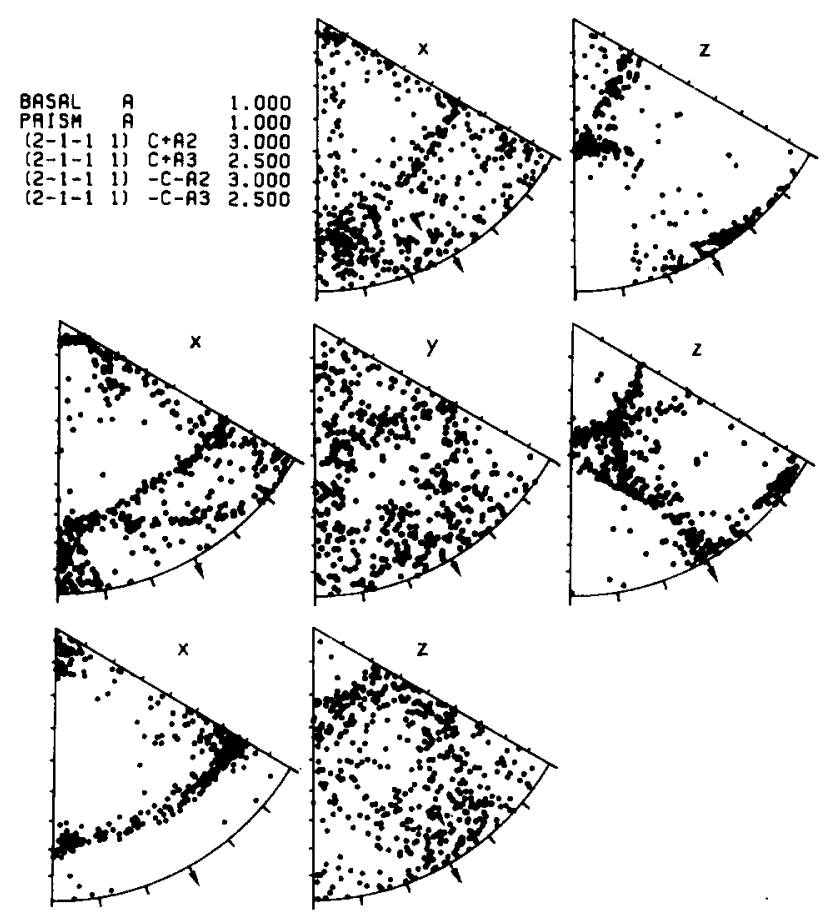

Fig. 3. Model quartzite $\mathrm{C}$ subjected to various progressive deformations. Details are the same as in Fig. 1.

progressive axially symmetric shortening; $c$-axes also form concentrations at high angles to $Z$. There are several classes of yield surface configurations that lead to such fabrics, produced in two categories: (a) simultaneous operation of basal $<a>$ and prism $<c>$ systems. In progressive plane strain for the situation where prism $<a\rangle$ glide is very soft, a $90^{\circ}$ crossed girdle of $\boldsymbol{c}$-axes results (Lister 1979) centred on $Y$ and resembling the fabric produced by Green et al. (1970) except for the strong concentration of $c$-axes parallel to $X$ in the simulated fabric. (b) operation of $\langle c+a\rangle$ systems with slip plane steeply inclined to the c-axis, for example the $\{2111\}$ or $\{2131\}$ systems. In progressive plane strain certain configurations give double maximum crossedgirdles of $\boldsymbol{c}$-axes but with strong concentrations of $\boldsymbol{c}$-axes parallel to $X$. Model $C$ was chosen from the latter class and produces a maximum of the positive rhombs, $r$, normal to the axis of shortening $Z$. Inverse pole figures are shown in Fig. 3.

All of these patterns deviate significantly from those observed in naturally deformed rocks. In particular, concentrations of $c$-axes parallel to $X$ are predicted by each model. This however is the 'kinking' or 'deformation band' orientation, where the crystal has the basal plane parallel to $Z$. For such orientations the $c$-axes move rapidly away from $X$ toward $Z$. If this behaviour could be included in the model, these concentrations at $X$ would disappear, and the orientations would migrate and accentuate other maxima in the fabric diagram, for example at $Y$.

\section{Mechanism activity}

According to the Taylor-Bishop-Hill analysis the amount of shear on a particular slip system, termed the mechanism activity, is determined by: (i) the imposed strain increment; (ii) the orientation of the strain increment axes relative to the crystal axes; and (iii) the other mechanisms that are operating to allow the strain increment to be achieved. There is no direct relation between the shear stress resolved on the glide plane in the slip direction, and the mechanism activity.

However, when the individual mechanism activity, $\gamma$, is plotted, there is a correlation between high mechanism activity and high Schmid factor (Fig. 4). This is because the equation for resolved shear stress:

$$
\tau=\sigma_{i j} \cdot \frac{1}{2}\left(n_{i} l_{j}+n_{j} l_{i}\right)
$$

has the same coefficients as the equation determining

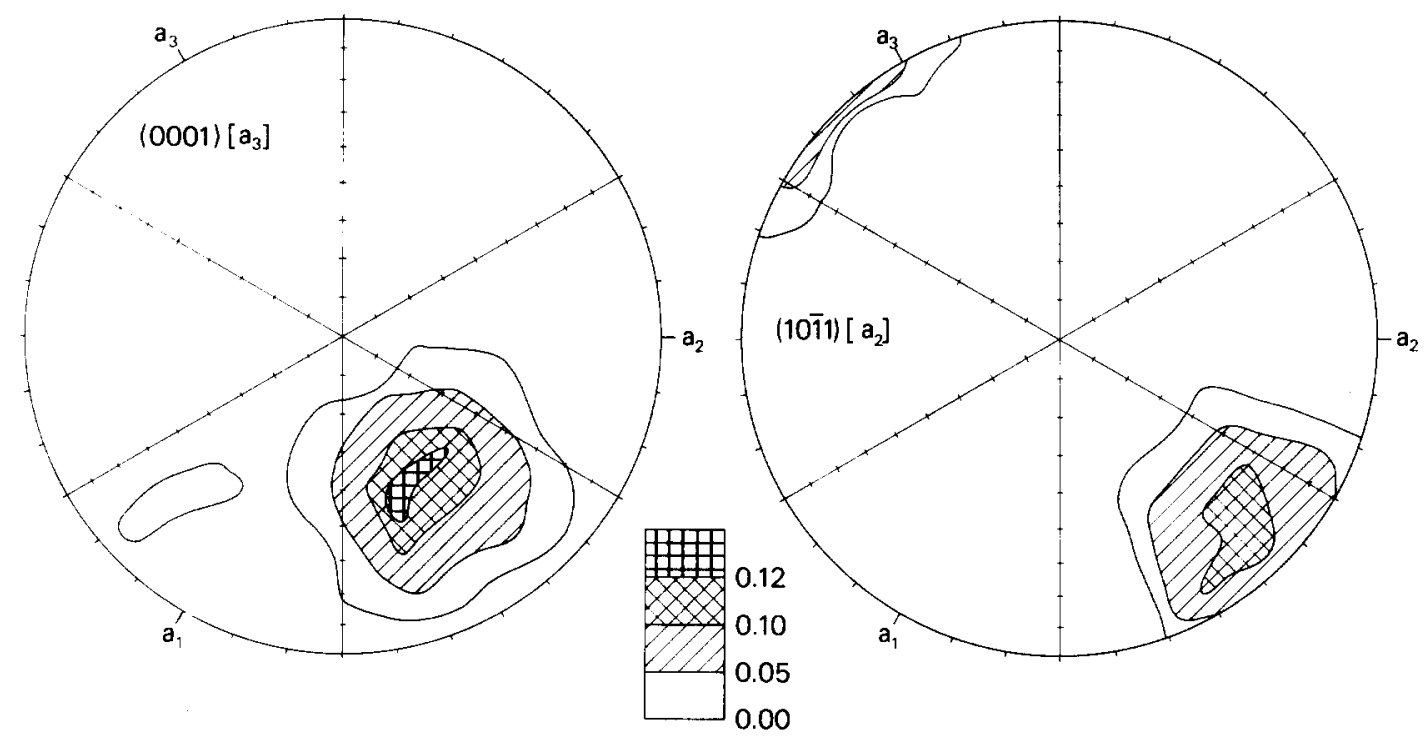

Fig. 4. Mechanism activity plotted with respect to crystal axes, model quartzite B. Left-hand diagram is for one basal (0001) $<a>$ system and right hand diagram is for one rhomb (1011) <a> system. Mechanism activity is plotted by marking shear strain caused where otherwise would be plotted the axis of shortening. Calculation is performed using a $10 \%$ axially symmetric shortening. Note there are 6 basal $<a>$ systems and 12 rhomb $<a>$ systems, and activity on these systems can be obtained by performing symmetry operations. Notice that mechanism activity in Taylor-Bishop-Hill theory has no direct relation to resolved shear stress, but high Schmid factors generally correlate with high mechanism activity. 


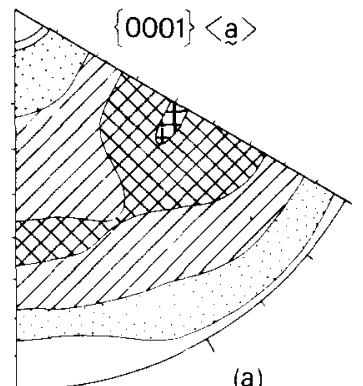

(a)

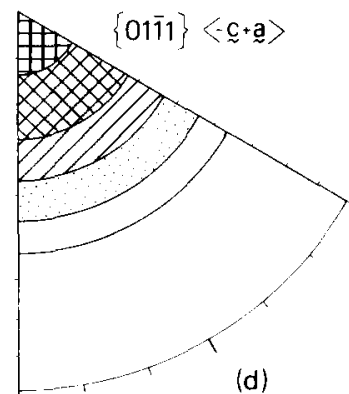

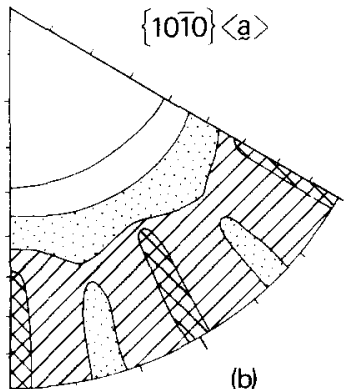

(b)

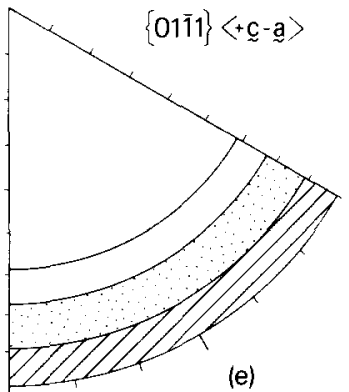

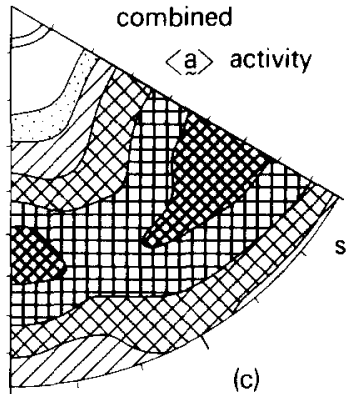

(c)

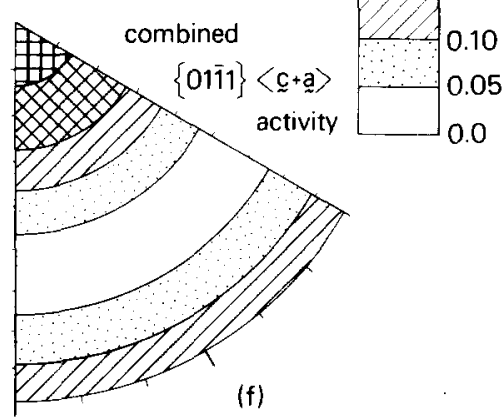

Fig. 5. Summary of mechanism activity for a $10 \%$ axially symmetric shortening imposed on a uniform population of grain orientations, with the yield surface configuration as for model quartzite A. Mechanism activity is plotted by adding the shear strains caused by operation of a particular group of mechanisms, and placing this value where otherwise would be plotted the axis of shortening, in the inverse pole figure (with reference to crystal axes). The c-axes is vertical, the a-axis is marked, and positive forms occur on the left-hand side of the primitive segment of the equal area upper hemisphere projection.
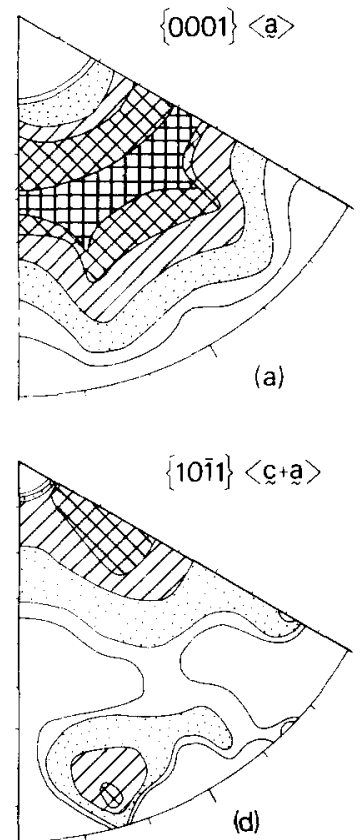
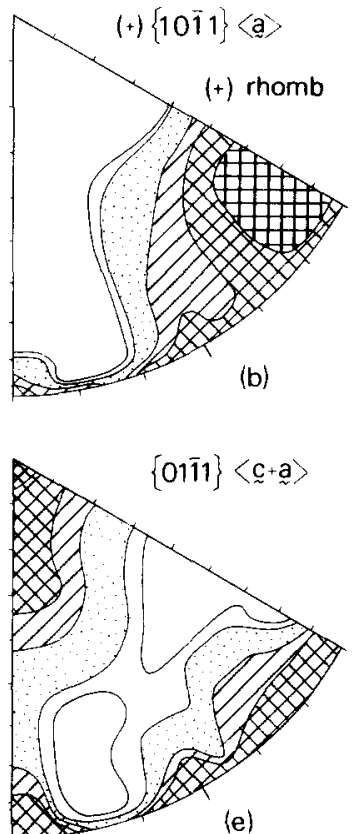

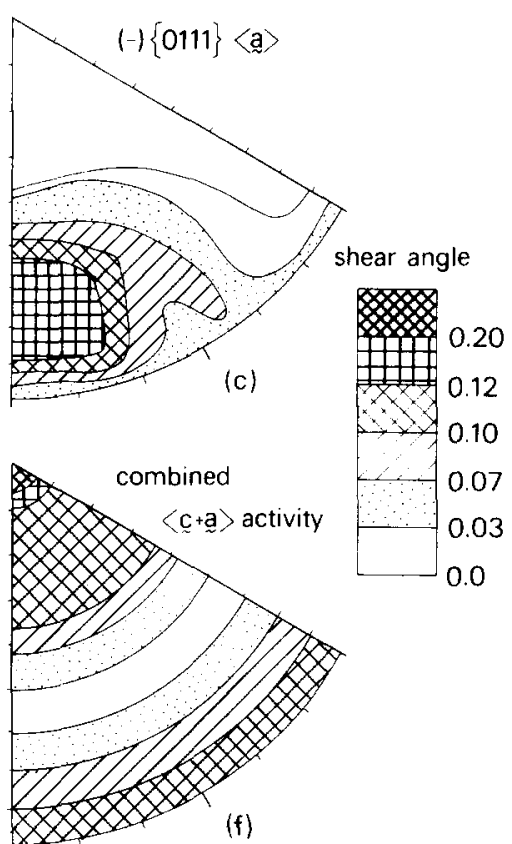

(f)

Fig. 6. Summary of mechanism activity for a $10 \%$ axially symmetric shortening imposed on a uniform population of grain orientations, with the yield surface configuration as for model quartzite B. Otherwise set out as for Fig. 5.

the strain increment, $\varepsilon_{i j}$, as a result of activity on the glide system (see Lister et al. 1978):

$$
\varepsilon_{i j}=\gamma \cdot \frac{1}{2}\left(n_{i} l_{j}+n_{j} l_{i}\right) .
$$

In the present application of the Taylor-Bishop-Hill theory we have used dislocation glide systems previously reported in the literature as operating during the experimental deformation of quartz. Figures 5 and 6 show mechanism activities on inverse pole figures for model quartzites $\mathrm{A}$ and $\mathrm{B}$. The following points arise. (i) The activity of glide systems with $\langle\boldsymbol{a}\rangle$ Burgers vector is generally dominant. (ii) The activity of glide systems with $\langle\boldsymbol{c}+\boldsymbol{a}\rangle$ Burgers vectors is generally subordinate, except for a few orientations such as $Z$ parallel to (0001) or $Z$ perpendicular to (0001), where the crystal is much harder (see Figs. 16 a \& e). The activity of the $\langle c+a\rangle$ systems is high in these orientations only because the homogeneous strain assumption requires hard grains to deform as much as soft ones, and thus obliges 'hard' systems to operate. (iii) The combined activity for $\langle\boldsymbol{c}+\boldsymbol{a}\rangle$ systems is simply related to the angle between the shortening axis and the $c$-axis. 


\section{THE EFFECT OF DEFORMATION HISTORY}

\section{Coaxial deformation histories}

The effect of various coaxial deformation histories has been explored for each model quartzite. Figures $7-9$ show $c$-axis pole figures on the lower hemisphere of an equal-area projection, for deformation histories that vary from progressive axially symmetric shortening to progressive plane strain and to progressive axially symmetric extension. The deformation gradients used are given in Table 3.

There are several points to note. (i) The strength of pattern concentration is a qualitative measure of the amount of strain. However, after large strain for models $\mathrm{B}$ and $\mathrm{C}$ a steady state situation is attained (Figs. 10 and 11). (ii) Pattern elements are visible after only $30 \%$ shortening. (iii) The skeleton of the fabric pattern, defined by the peaks, crest and ridge-lines of the concentration pattern, is symmetrically related to the finite strain axes. (iv) Once the skeleton outline of a pattern is defined there are no changes in its orientation relations with the strain-axes as strain increases. For example the opening angle of $c$-axis girdles (Fig. 10) becomes more accurately defined as the girdle becomes more intensely populated, but the opening angle does not increase with increasing strain. (v) The fabric indi- cates the position of the finite strain axes, in that small circle girdles develop about $Z$, and cleft girdles form about $X$; girdles cross on the $Y$-axis and invariably lie at high angles to $X$ for progressive plane strain. Commonly a $c$-axis maximum forms at $X$, but a large pole free area surrounds $X$, so it is possible to determine directly the axis of extension.

\section{Non-coaxial deformation history}

As an example of a non-coaxial history this paper will be restricted to discussing the results for progressive simple shear. Other non-coaxial histories have been discussed in Lister \& Price (1978). The c-axis fabrics developed during progressive simple shear for each model quartzite are portrayed in Figs. 12-14. Figure 15 shows $a$-axis pole figures for a progressive simple shear involving 50\% shortening, and a-axis figures for the corresponding coaxially applied progressive plane strain. The following characteristics are to be noted

(i) Pattern concentrations become more intense with increasing strain, though for model B note that after a shear strain of about 3 , a steady state concentration is attained.

(ii) Definite asymmetry appears in the orientation distribution and in the skeletal outline of the fabric after

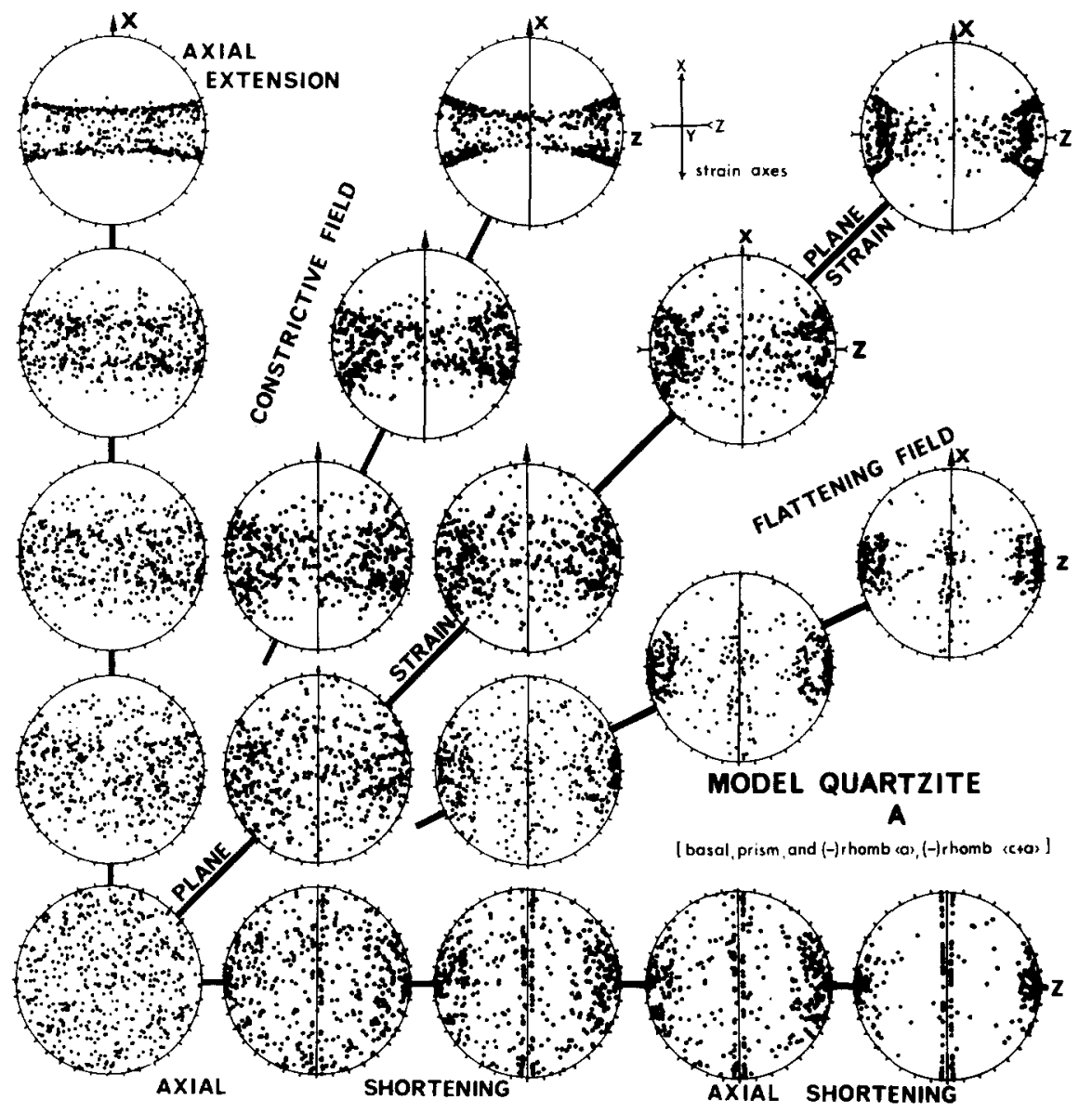

Fig. 7. The effect of different coaxial deformation histories for model quartzite A. c-axis fabrics are shown on a Flinn diagram. Five different strain histories are portrayed (see Table 3 for the deformation gradients applied in each history). Fabrics are shown on the lower hemisphere of an equal-area projection, and diagrams are portrayed for fabrics after 10,14 , 18 and 31 increments. For plane strain and axial shortening this involves respectively $40 \%, 50 \%, 60 \%$ and $80 \%$ overall shortening. The original random $c$-axis fabric is shown at the origin. Each pole figure involves $500 \mathrm{c}$-axes. 


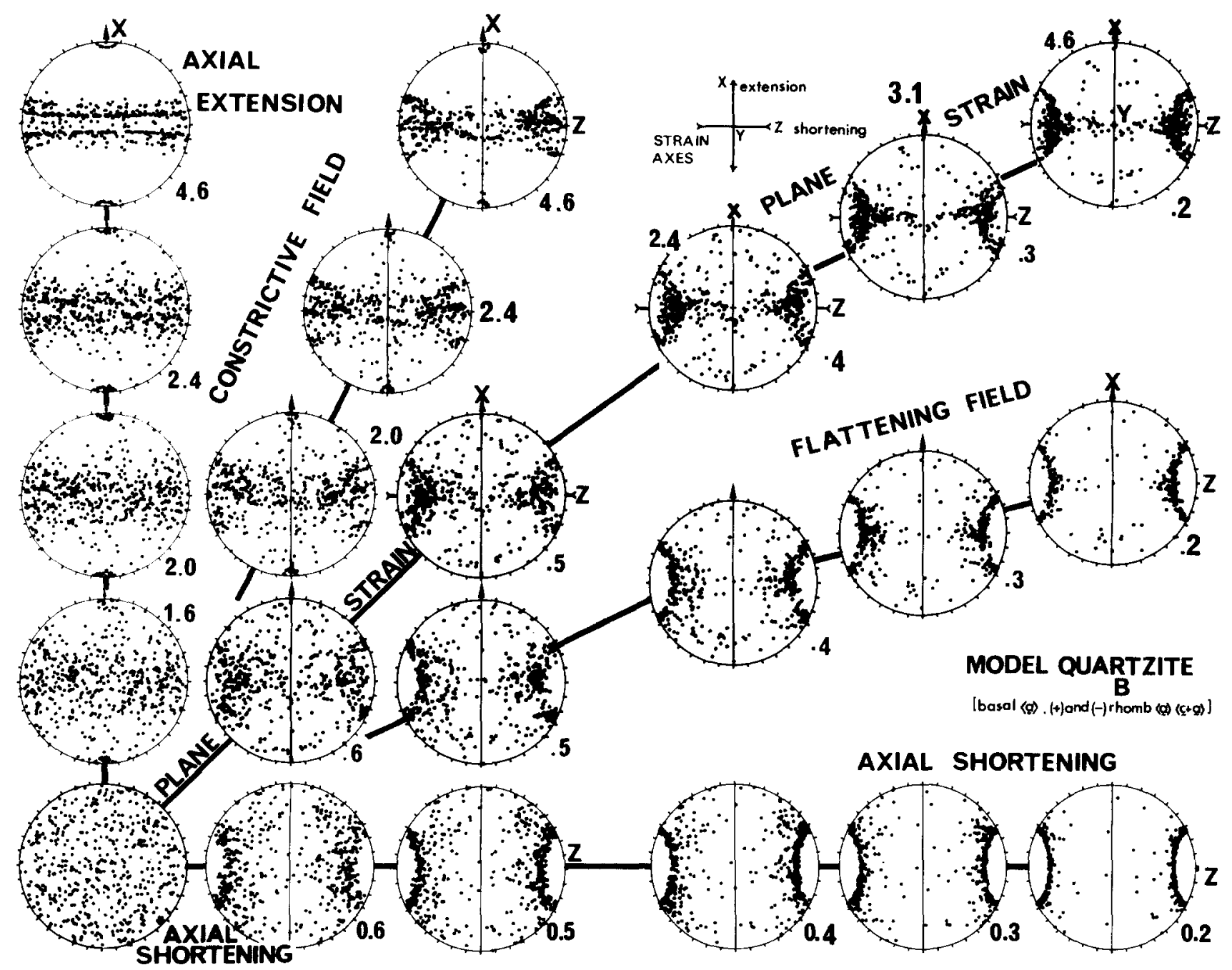

Fig. 8. The effect of different coaxial deformation histories for model quartzite B. Details are as in Fig. 7. Some of the principal stretches are shown besides the diagrams, namely overall extension in the constrictive field, and overall shortening in the flattening field. The fabric variation shown here occurs in nature. Cleft girdles form in quartzites for which a cigarshaped finite strain ellipsoid is appropriate, double-wedge or crossed-girdle fabrics form in plane strain, and small-circle conical distributions result from coaxial deformation involving pancake shaped finite strain ellipsoids.

axial extension plane strain axial shortening

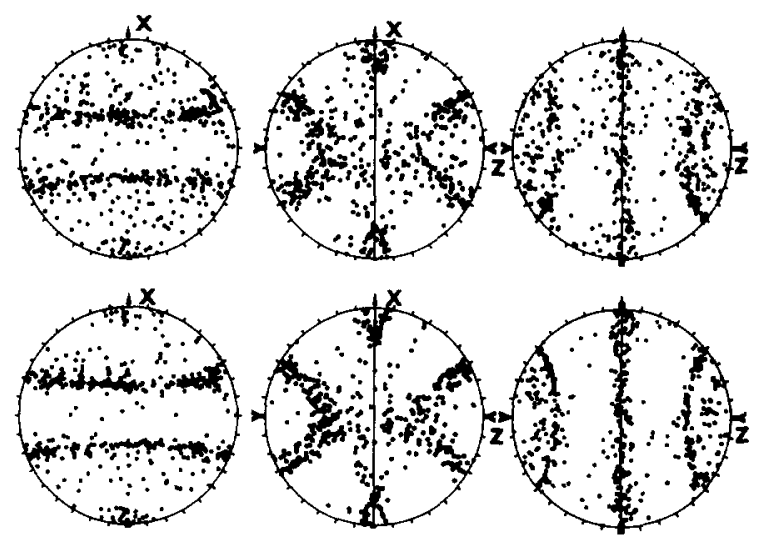

Fig. 9. model quartzite $\mathrm{C}$ gives rise to these $\mathrm{c}$-axis fabrics when subjected to axially symmetric extension (left column), plane strain (middle column) and axially symmetric shortening (right column). Pole figures shown for 7 increments (top row) and 14 increments (bottom row) using the deformation gradients shown in Table 3 (involving respectively 30 and $50 \%$ shortening for plane strain). It will be noted that model $\mathrm{C}$ develops fabrics faster than did the other model quartzites. Note that many 'grains' are rotated so that the $c$-axis is close to the extension axis. This is the 'kinking' orientation since the basal plane is oriented near to parallel with the shortening axis. If kinking was included in the model the maximum near $X$ would disappear. a certain shear strain. At low shear strain the asymmetry is not marked, and it would be possible to ascribe an orthorhombic symmetry to the fabric skeleton, and to state that it was symmetrically related to the finite strain axes; at high shear strains the fabric skeleton attains a characteristic asymmetry which can be used to deduce the sense of shear.

(iii) The fabric asymmetry is always such that the caxis concentrations have a leading edge which is sharply defined, and it is orthogonal both to the flow plane and the flow direction. A significant number of basal planes align themselves precisely in the flow plane (this tendency is particularly marked for models A and C).

(iv) Models $\mathrm{A}$ and $\mathrm{C}$ develop a c-axis girdle precisely orthogonal to the flow plane and the flow direction. This tendency occurs also for model B but is not as marked. (v) The a-axis fabric develops an asymmetry which can be used to deduce the sense of shear. The greatest concentration of $a$-axes is in the flow plane parallel to the flow direction.

(vi) Since the strongest a-axis maximum defines the flow direction, the angle between this maximum and the $X$-direction can be used to estimate the shear strain. 

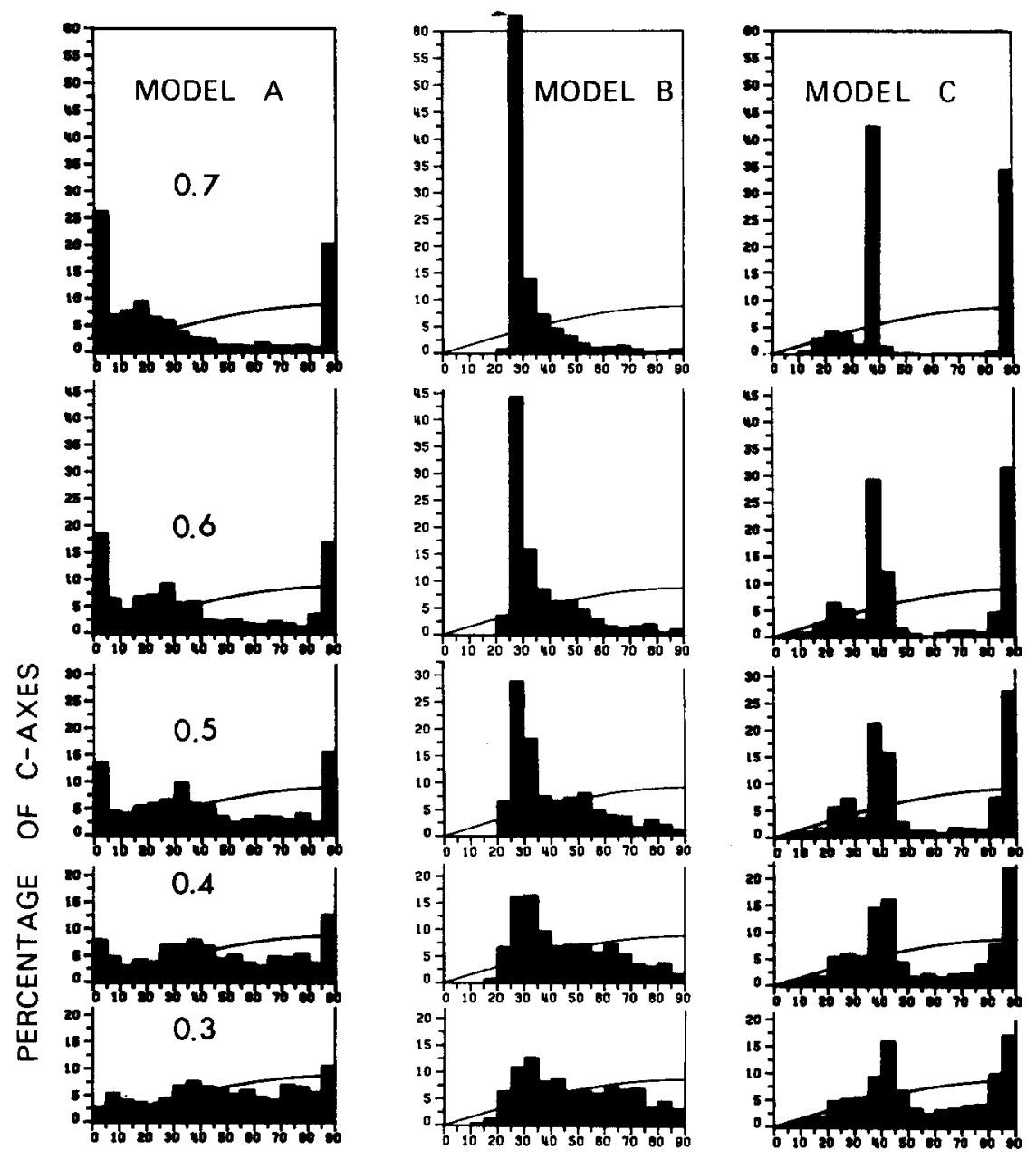

ANGLE WITH THE AXIS OF SHORTENING

Fig. 10. Histograms show angular distribution of $c$-axes with respect to the axis of shortening. Each of the model quartzites is portrayed after $7,10,14,18$ and 23 increments of a $5 \%$ axially symmetric shortening, involving overall shortening as shown $30,40,50,60$ and $70 \%$ respectively. Note that the opening angle of the small-circle girdles does not change with increasing finite strain, but the pattern concentrations become more intense. The sine curve represents the distribution that would be obtained if the pattern was completely uniform. Tullis et al. (1973) present data that is normalized with respect to this curve.

(vii) The skeletal outline of the c-axis fabrics is evident after low shear strains, and once the fabric skeleton is defined it remains in a constant orientation relative to the kinematic framework. That is, the fabric does not rotate with the finite strain axes, but is more or less independent of them. The fabric remains stationary with respect to the flow plane and with respect to the instantaneous stretching axes.

Some other additional points are illustrated by Fig. 16 which shows work maps and reorientation trajectories for the three model quartzites. Work maps are prepared by calculating the work done in deforming a crystal by a $10 \%$ axially symmetric shortening, in various orientations, and then plotting the result where otherwise would be plotted the axis of shortening on an inverse pole figure (with respect to crystallographic coordinates; c-axis vertical, a-axis marked, using a segment of the upper hemisphere equal-area projection, positive forms on the left side of the segment). A work map can also be interpreted in terms of heat dissipation rate if one considers each increment to be performed in constant time. The work maps are shown in Figs. 16(a), (e) and (i) and the remaining diagrams represent the reorientation trajectories. A $10 \%$ axially symmetric shortening is imposed on a collection of grains and the reorientation calculated. The tangents to the reorientation trajectories are shown (Figs. $16 \mathrm{~b}, \mathrm{f} \& \mathrm{j}$ ) on an inverse rotation diagram (this shows the reorientation of the axis of shortening). The reorientation trajectories can be more clearly visualized by showing tangents to the trajectories for a randomly oriented collection of $\mathbf{4 0 0}$ grains after they have been subjected to $20 \%$ shortening. An inverse pole figure is presented for each model quartzite (Figs. $16 \mathrm{c}, \mathrm{g} \& \mathrm{k}$ ) showing the tangent to the reorientation trajectory for the axis of shortening, and a pole figure (Figs. $16 \mathrm{~d}, \mathrm{~h} \& \mathrm{l}$ ) shows the tangent to the reorientation trajectory for the $c$-axis.

The following features are brought out by Fig. 16 .

(i) Specific initial orientations rotate during deformation to specific final orientations along specific reorientation trajectories. It is common for grain orientations to migrate rapidly toward girdles and maxima, the rate of rotation decreasing as these pattern elements are occupied. Orientations then migrate along 
MODEL A
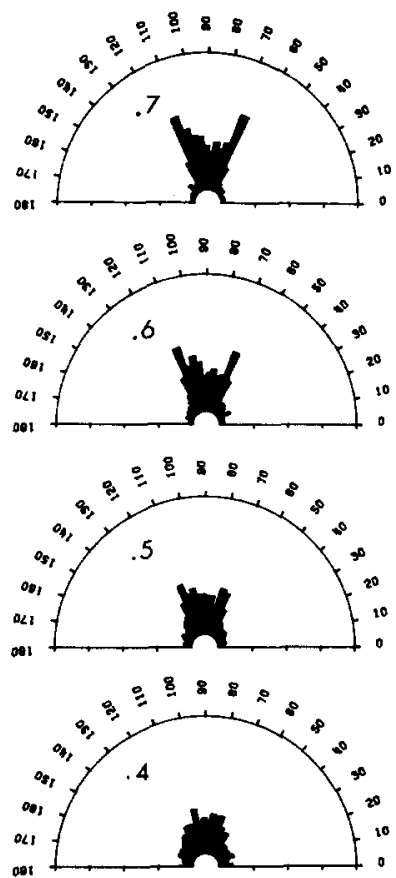

MODEL B
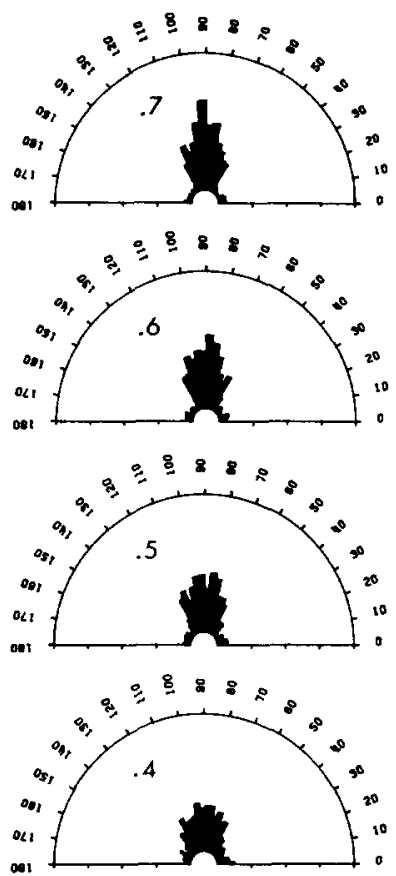

MODEL C
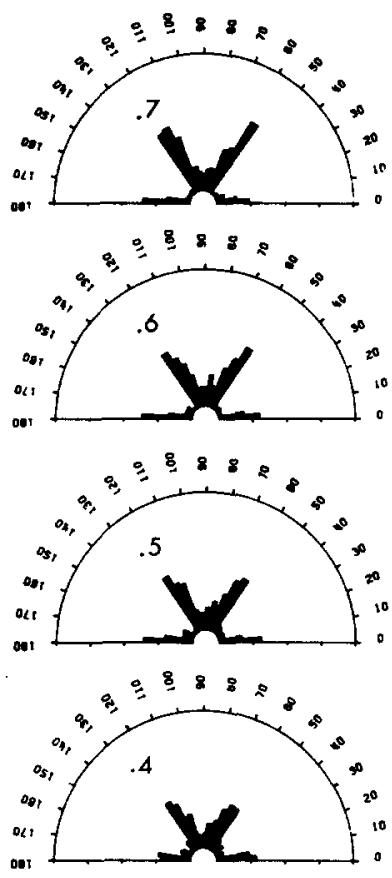

Fig. 11. Rose-diagrams show angular distribution of the trend of the $c$-axes in the $X Z$ plane of the finite strain ellipsoid, with each of the model quartzites subjected to $10,14,18$, and 23 increments of a plane strain deformation (Table 3). This involves shortening as shown. The plane of flattening is E-W. Graduation marks indicate $5 \%$ of the population. Note that Model quartzites A and B develop double wedge patterns in plane strain. However, in Model A no pole free area develops at $Z$ (the axis of shortening) and the strongest two maxima form where the pattern intersects the $X Z$ plane (similarly model C). However model B forms two maxima $90^{\circ}$ rotated to these maxima, forming concentrations where the arms of the crossed-girdle intersect in the $Y Z$ plane. This variation can be seen in the rose diagrams. Note also that opening angles do not change with increasing strain, but pattern elements become more intensely defined.
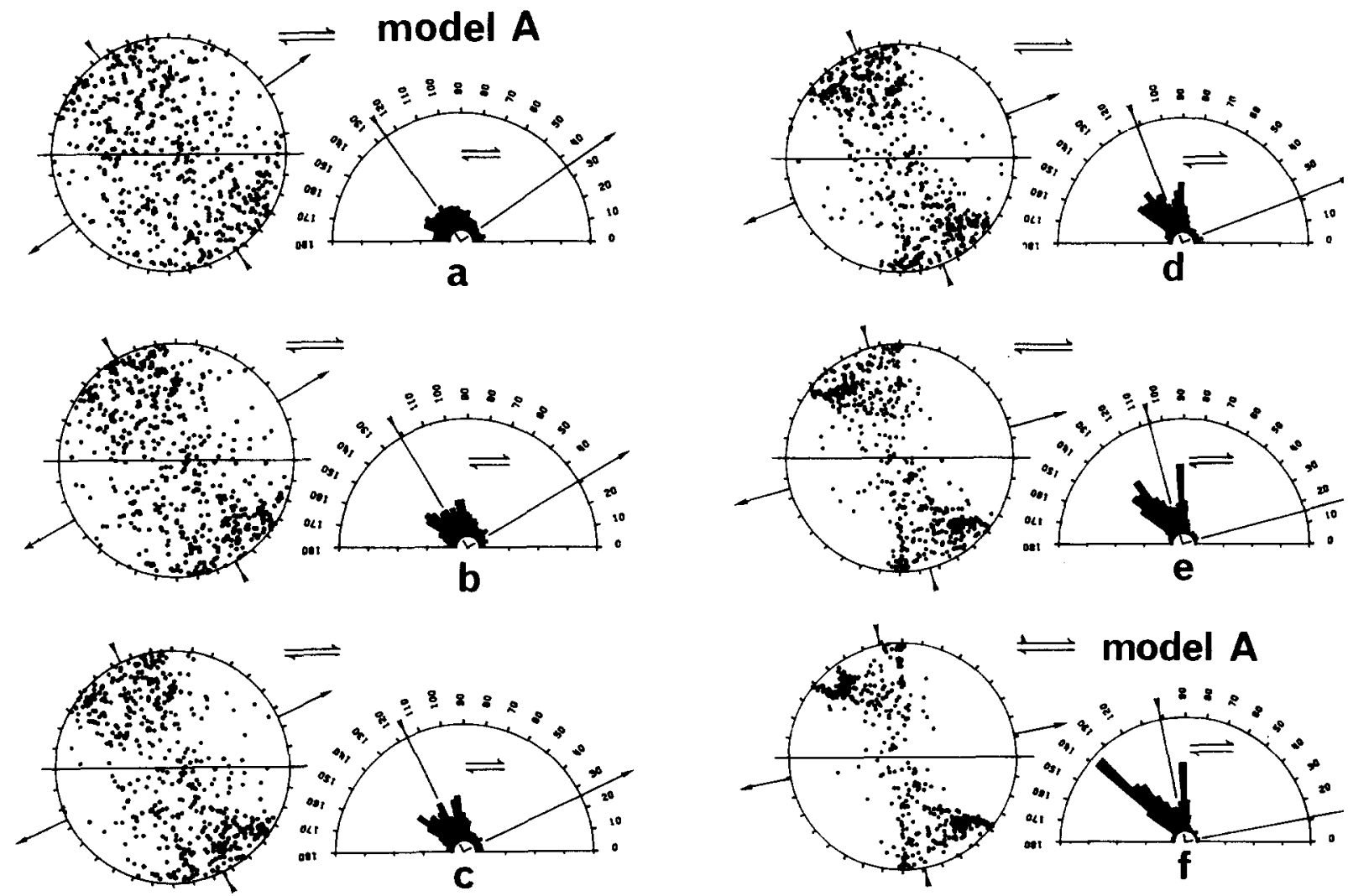

Fig. 12. Model quartzite $A$ is subjected to progressive simple shear, and resulting $\mathbf{c}$-axis fabics are shown after shear strains of $0.7,1.0,1.5,2.1,3.0$ and 4.0 ; involving $30,40,50,60,70$ and $80 \%$ shortening. Increments involving 0.1 shear strain are used. The rose diagrams show the trend of the $c$-axes in the $X Z$ plane, and the asymmetry of the fabric is thus emphasized. Note the girdle of $c$-axes that develops is orthogonal to the shear direction. Graduation marks indicate $5 \%$ of the popula- 

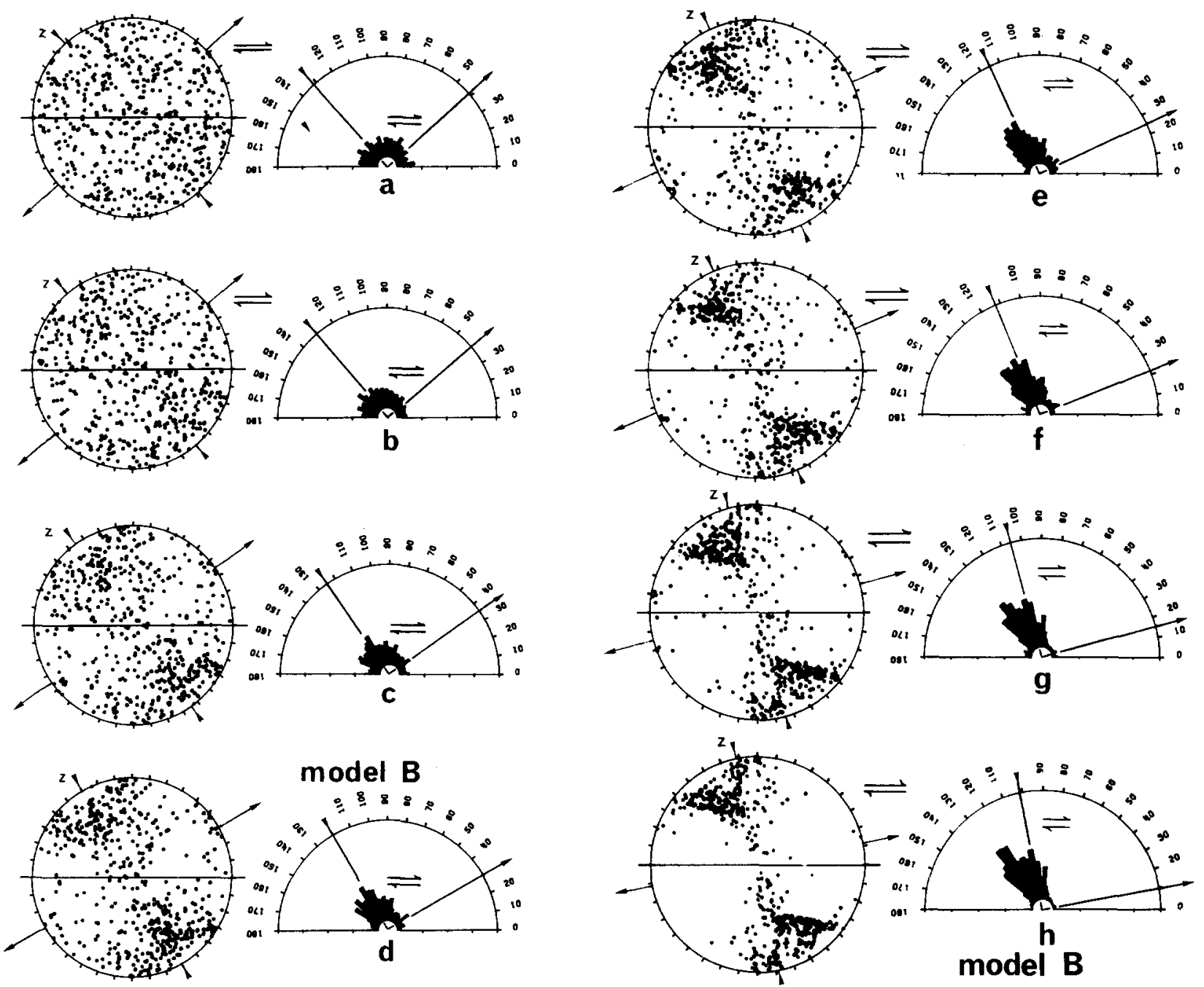

Fig. 13. Model quartzite $B$ is subjected to progressive simple shear and $c$-axis fabrics portrayed for shear strains $0.2,0.4,0.7$, $1.0,1.5,2.1,3.0$ and 4.0 but otherwise as for Fig. 12. Note that as the fabric develops it does not rotate to keep track with the finite strain axes, but remains fixed with respect to the kinematic framework, e.g. pattern elements remain constant in orientation with respect to the flow plane and flow direction. Again one girdle is orthogonal to the flow direction.

the girdles at a slower rate toward the maximum. Thus, with increasing strain, certain areas on the $c$-axis pole figures rapidly depopulate to form a pattern of maxima and connecting girdles, defining families of orientations. The pole free areas have as much significance as do the maxima and girdles. With increasing strain the girdles tend to depopulate in favour of the maxima. An important comment on statistics is that submaxima are not usually clearly and reproducibly defined until more than 400 grains are plotted (cf. Nicolas \& Poirier 1976, pp. $314-318$ ).

(ii) On the pole figures certain orientations are populated with increasing concentrations of c-axes as deformation proceeds. Specific areas can be delineated as the source areas that provide the initial orientations for developing maxima and girdles. These source areas depopulate to form pole free areas. Since the relative extent of the source area determines the strength of the maximum, preferred orientation is affected by initial orientation distribution. Peculiar symmetry effects will result as a consequence of non-random starting populations (Lister \& Price 1978). The maxima and girdles still form in the same orientations, but the intensity distribution over the fabric skeleton is different. (iii) Intensities are higher in the simulated fabrics than in naturally and experimentally produced fabrics. This is probably mostly a function of the assumption of homogeneous deformation incorporated in the model. The simulation produces mathematically exact maxima, which are precluded by inhomogeneous deformation in reality. Also, recrystallization processes serve to continuously modify a developing deformation fabric in many natural situations. Note also that intensity distribution depends on the measuring process, the contouring process, and commonly on the number of grains measured.

\section{DISCUSSION}

The necessary and sufficient assumptions made in the application of the Taylor-Bishop-Hill analysis are as follows. (i) The material behaves in a rigid-plastic fashion. (ii) Dislocation systems interpenetrate without interference (i.e. system activities are mutually independent and not coupled). (iii) The deformation caused by one glide system can be accurately approximated as a continuous and homogeneous simple shear during each 
increment. (iv) All deformation takes place by dislocation glide and there is no grain boundary sliding. (v) Deformation is homogeneous on the grain scale.
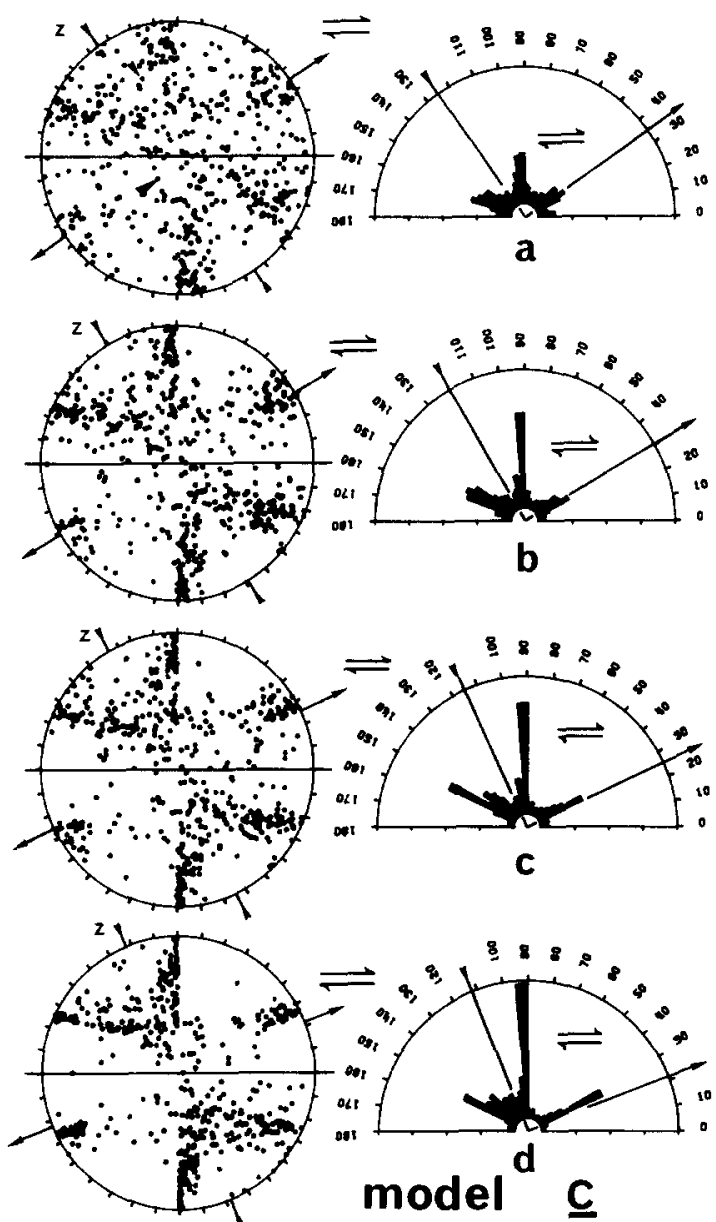

Fig. 14. Model quartzite $C$ is subjected to progressive simple shear, and $c$-axis patterns portrayed for progressive simple shear, with shear strains $0.7,1.0,1.5,2.1$ involving $30,40,50$ and $60 \%$ shortening respectively. Again the pattern is asymmetric and the sharpest and most distinct girdle is perpendicular to the flow direction. The strongest maximum disposes many 'grains' so that the basal plane lies in the flow plane, and the $a$-axis lies parallel to the flow direction.
These are simple approximations to the complexity observed in naturally and experimentally deformed quartzites. However, the Taylor-Bishop-Hill theory has provided the basis for a versatile model allowing simulation of the development of crystallographic fabrics during plastic deformation and this model has been used to develop some understanding of the effect of deformation history on fabric development. The most important general results to arise from the application of this model are as follows.

(i) There is an overall variation of c-axis fabric type with the nature of the strain increment, so that fabrics can be used to indicate whether the deformation history was in the field of progressive plane strain, constriction, or flattening.

(ii) The fabric orientation and characteristics are determined by the kinematic framework on the scale of the hand specimen and not by the orientation of the principal axes of finite strain.

(iii) Specific initial orientations reorient towards specific end-orientations and a fabric skeleton can therefore be defined more or less independently of the orientation distribution once a fabric is strongly enough developed.

(iv) The closing stages of crystalline plastic deformation can exert a profound influence on fabric development.

These hypotheses form the basis of a kinematic theory of fabric development and can be tested quite independently of the validity or relevance of the strict Taylor-Bishop-Hill model.

In the remainder of this section we discuss two aspects of fabric interpretation that have played a dominant role in discussions of fabric diagrams over the past fifty years. These are the significance of symmetry arguments and the role of the kinematic framework (or 'movement picture') in the interpretation of fabrics.

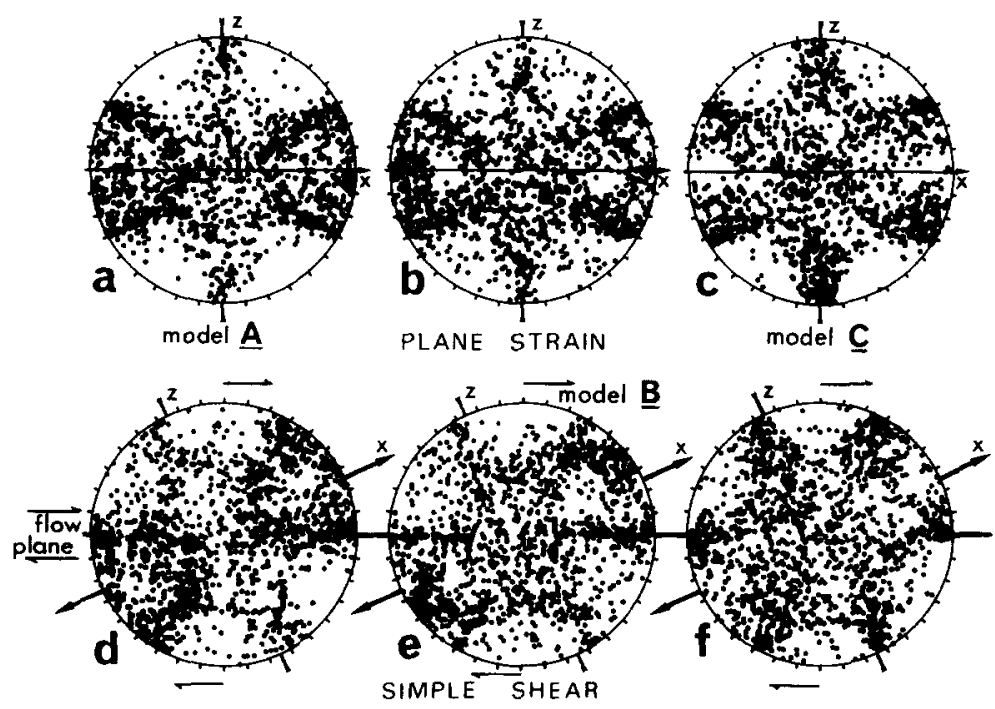

Fig. 15. The a-axis fabric patterns for plane strain (top row) and for simple shear (bottom row) are shown for each model quartzite. In all cases $50 \%$ shortening is involved. For simple shear the strongest concentration of $a$-axes occurs parallel to the flow direction. 

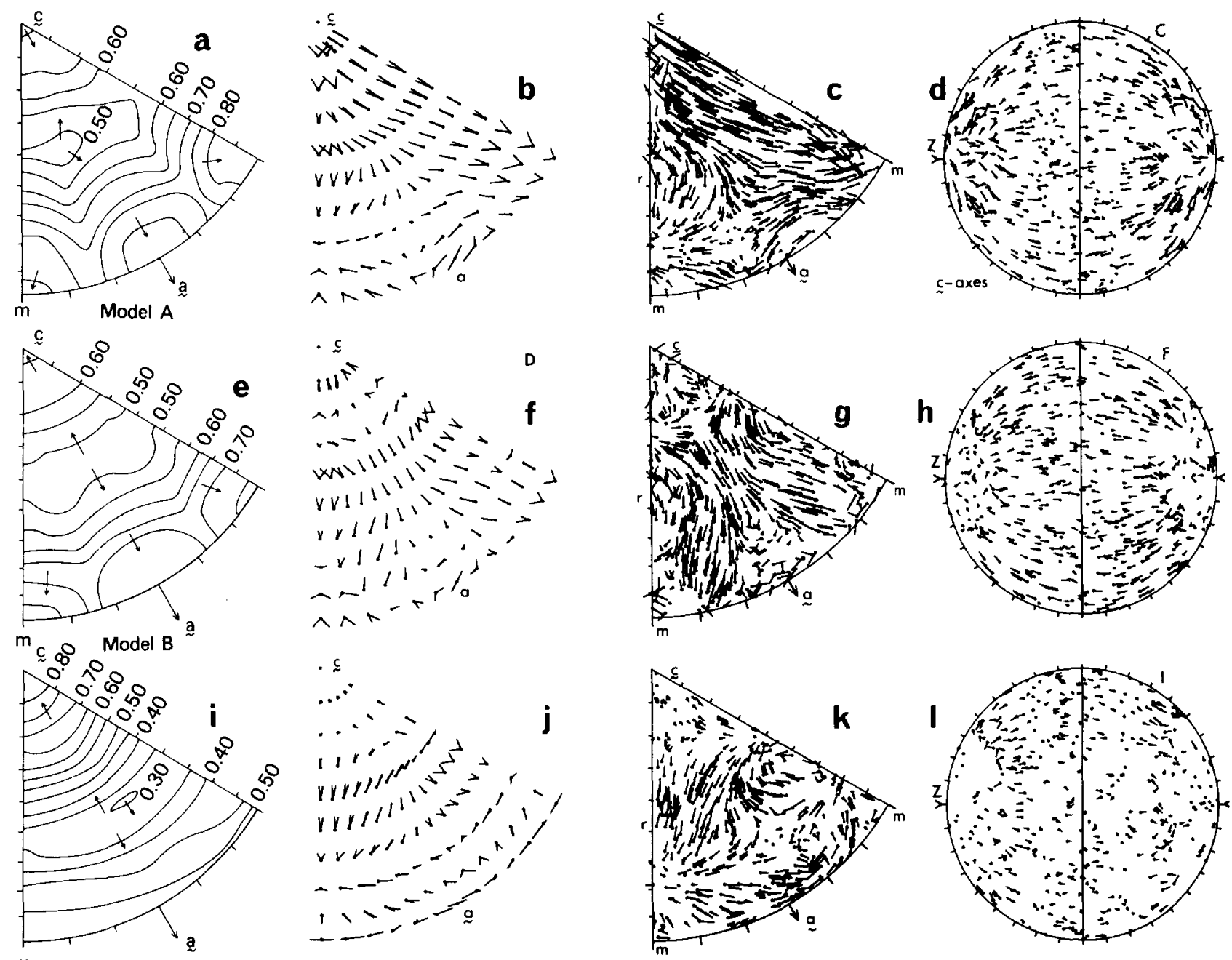

Fig. 16. Work maps and reorientation trajectories for the three model quartzites; A (top row), B (middle row) and C (bottom row). Work maps are shown in $a, e$ and $i$. The remaining diagrams are reorientation trajectories. See text for discussion.

\section{The symmetry principle}

The symmetry principle used extensively by Sander and subsequent workers was formally enunciated by, and its use in structural geology clarified by, Paterson \& Weiss (1961). It states that: "the point group symmetry of the effects is not less than the common symmetry of the point group symmetry of the causes". Thus, the absence of a symmetry element in the point group symmetry of the effects implies the absence of that symmetry element in one of the point symmetry groups of the causes. Considerable care must be exercised in applying this principle to deformed rocks because additional fabric elements can usually be found and added to the list of possible causes reducing eventually the common point group symmetry to triclinic. For example, it might be shown that the overall symmetry in a specific situation is triclinic, although the developed quartz fabric has intrinsically higher symmetry. In situations such as this, the factors that reduce the overall symmetry of the situation must be examined carefully before they are added to the list of causes contributing toward the crystallographic fabric. It might be, for example, that an observed foliation induced virtually no strength aniso- tropy during the period of deformation when the quartz fabric formed, and thus did not influence the system. Although such arguments cannot be justified by using the symmetry principle, some explanation should be sought if an effect has higher than the common symmetry of the listed causes. It may be that some items in the list are not relevant.

Applying the symmetry principle leads to the conclusion that asymmetric fabrics can arise in three ways.

(i) If the initial orientation population did not contain a sufficient scatter of orientations, or if it was not random. In many cases quartz aggregates arise from the deformation and recrstallization of a few large grains. Recrystallization and deformation may scatter orientations sufficiently to over-ride this effect, which otherwise has the same result as the existence of an earlier fabric. Initial fabrics of sedimentary or early tectonic origins are not uncommon.

(ii) If the kinematic description of the deformation did not remain constant. In such complex deformation histories, the asymmetry of the fabric need not be related to 'sense of shear', and need not imply a non-coaxial deformation history. 

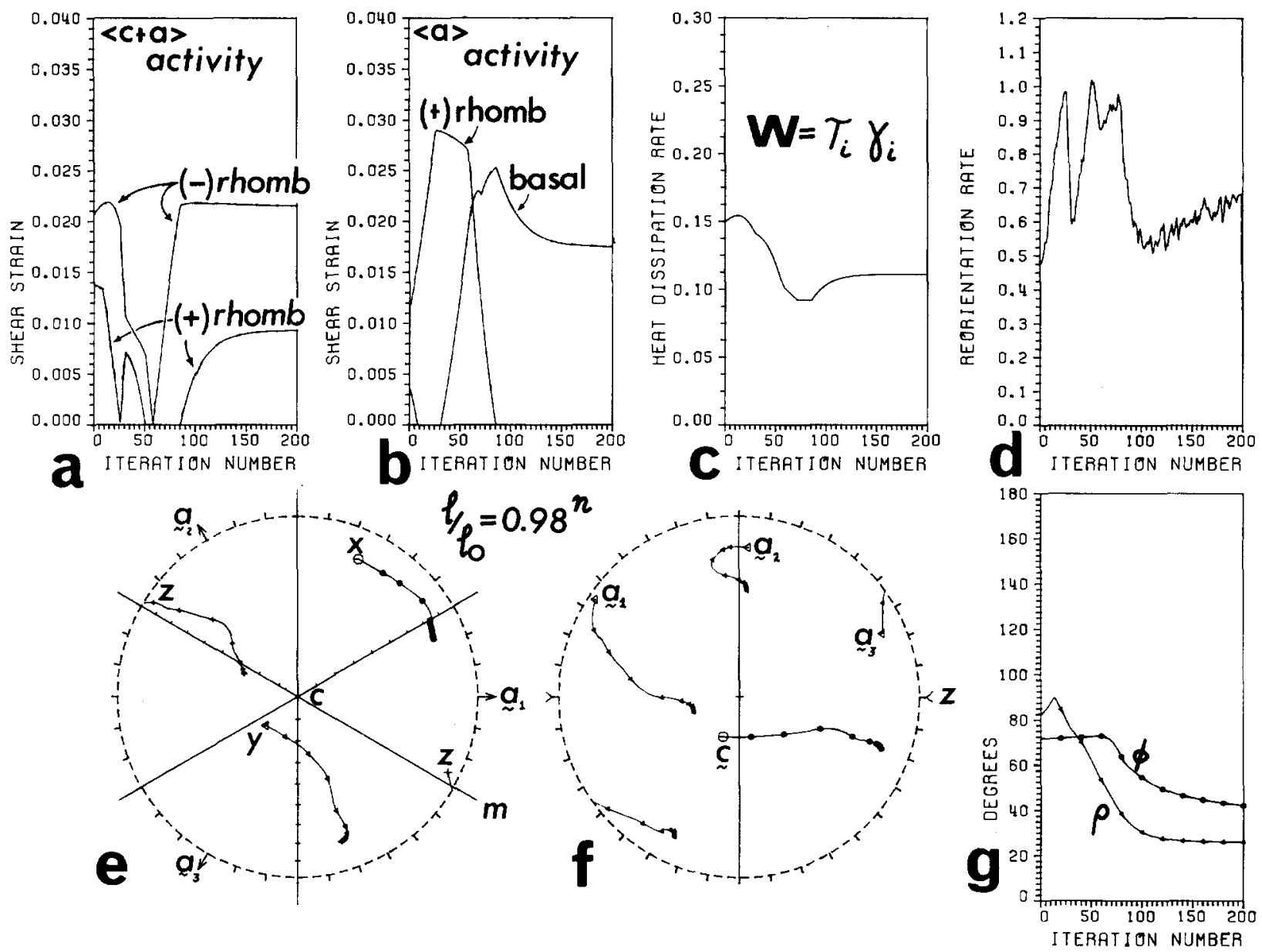

Fig. 17. Aspects of the reorientation of a single grain subjected to 200 increments of a $2 \%$ axially symmetric shortening. Model quartzite B. See text for details of figure and for discussion.

(iii) If the kinematic description of the deformation was constant, but the deformation history was noncoaxial.

Only for case (iii) can it be argued that an asymmetry in the fabric is directly related to a non-coaxial deformation history such as progressive simple shear, and the fabric asymmetry used to infer a 'sense of shear'.

Role of the kinematic framework in determining fabric development

The simulation studies suggest that the kinematic framework plays a role of hitherto unsuspected importance in determining the nature of a pattern of preferred orientation. If the kinematic description does not change throughout a deformation history then the instantaneous stretching axes remain constant in orientation. Crystalline grains tend to rotate toward preferred orientations such as small-circle girdles or maxima. The orientation of these elements does not alter during deformation with respect to the kinematic framework.

The effect of continuing deformation is merely to increase the intensity of concentrations on the fabric diagram. No case has yet been found in which the early formed orientation relations of the pattern elements alter with respect to the kinematic framework. This is an important point. Note however that some girdles of $c$ - axes appear to increase their opening angle with continuing deformation. During simulation of the development of a $40-50^{\circ}$ girdle of $c$-axes for progressive axially symmetric shortening, a pole free region forms at the axis of shortening in the early stages of deformation and expands with increasing strain. Increasing deformation increases the intensity of the girdle and decreases its effective width.

The results above are significant for non-coaxial histories where the stretching axes do not coincide with the finite strain axes, and the finite strain axes are continuously rotating with respect to the kinematic framework. According to the results obtained here, the developing fabric does not track the orientation of the axes of finite strain, rather, maxima develop with specific angular relations to the kinematic framework. The fabric reflects the position of the finite strain axes in only an incidental fashion, in as much as it is determined by the relation of the finite strain axes to the deformation history as a whole.

The rotation of a grain toward rotationally stable end positions is initially quite rapid if the orientation of the grain differs considerably from that position. This is illustrated in Fig. 17 where one interesting initial orientation is selected for model quartzite $B$ and subjected to 200 increments of a $2 \%$ axially symmetric shortening. Finite shortening can be calculated by applying the exponential rule shown. The computer 
program plots: (a) the shear strain caused by the activity of $\langle c+a\rangle$ systems per increment; (b) the shear strain caused by the activity of $\langle a\rangle$ systems per increment; (c) work done or heat dissipated per increment (arbitrary units); (d) reorientation of the c-axis per increment (in degrees); (e) the reorientation trajectories for the principal axes of finite strain $X, Y$ and $Z$, plotted on the upper hemisphere equal-area projection, with respect to crystal axes; (f) the reorientation trajectories of the $c$ axis and the $a$-axes plotted with respect to the deformation axes; and $(\mathrm{g})$ the change of spherical coordinates of the $c$-axis, with $\varphi$ the trend of the $c$-axis on the plane normal to $Z$, and $\rho$ the angle between $c$ and $Z$. The trajectories are marked every 20 iterations. A time scale of one increment per million years implies an approximate strain rate of $10^{-15} \mathrm{~s}^{-1}$. Note that the crystal axes reach a rotationally stable end orientation after 90 increments, and that orientation softening reduced the dissipation rate by a factor of one third during this time. If such a phenomenon occurs in natural rocks it could be an alternative to the often-invoked strain softening mechanisms, used to explain narrow high strain zones. Once the end-orientation is attained, with the c-axis about $25^{\circ}$ from the axis of shortening, and even as the end-orientation is approached, activity builds up on the hard $<c+a>$ glide systems, and orientation hardening takes place. If latent hardening assumptions are built in to the model (see Lister et al. 1978) the crystal axes would gyrate continuously and would swivel and rotate around the axis of shortening. In the case reported here no latent hardening was considered so the crystal axes rotate a degree or so, backwards and forwards, in a rotationally stable orientation after sufficient strain has been imposed.

Even though the initial reorientation of a grain may be quite rapid, as the crystal axes approach the endorientation, activity on other systems has the effect of reducing the rate of reorientation. This has important consequences if the deformation on the grain scale changes during the deformation history, for example, if there is a change in the orientation of the instantaneous stretching axes with respect to the already developed fabric. The grains will find themselves differently oriented to the kinematic framework and begin migrating toward end-orientations defined with respect to the new positions of the stretching axes. A pre-existing fabric will be reoriented to reflect the influence of the change in the kinematic framework, although the girdles and maxima that define the elements of the pre-existing pattern may still be preserved in different orientations. Maxima will migrate to new orientations, girdles will expand or contract or undergo distortion, and the pattern will be generally transformed. However, unless the pattern was reasonably well developed before the change a certain disintegration occurs. The older pattern elements modify as well as migrate and earlier fabrics can be quickly obscured.

These results follow because the lattice rotation is determined by factors including the orientation of the grain and the nature of the deformation increment. The previous deformation history has no bearing on the matter, except in a statistical way in determining grain orientation distribution. Thus, the closing stages of the deformation history can be of considerable importance in influencing fabric development.

\section{CONCLUSIONS}

The fabric simulations reported here indicate that crystallographic fabrics developed in plastically deformed quartzites may allow information to be obtained about the type of strain history, the total strain, and the orientations of both the (kinematic) stretching axes and the principal axes of finite strain.

For example, in movement zones developed by progressive simple shear, it may be possible to determine the sense of shear from the asymmetry of the fabric skeleton. The differences between fabrics simulated for progressive plane strain (coaxial) and for progressive simple shear are important in this regard. The noncoaxial history produces similar fabrics to those developed in the coaxial case, but a distinct asymmetry develops in the fabric skeleton at high strains for the non-coaxial history. The flow plane, flow direction and sense of shear are then immediately evident. However for natural fabrics to be interpreted in this way, the deformation history has to have been relatively simple, and there should be no effects relating to the orientation distribution of crystal axes prior to deformation.

In coaxial deformation histories, the type of strain increment can be decided by inspection of the fabric, whether it be axial shortening, plane strain, axial extension, or something in between. The orientations of the principal axes of finite strain are also evident since they are symmetrically related to the fabric skeleton.

Fabrics develop in relation to the kinematic framework. The pattern elements that define the fabric skeleton form in specific orientations relative to the attitude of the instantaneous stretching axes. These relationships remain constant with increasing strain except that the pattern concentrations become more intense. If the type of deformation changes during the deformation history the developing fabric pattern will attempt to track the changing orientations of the kinematic framework.

In the simulations the amount of strain is indicated by the degree of preferred orientation since this steadily increases with strain. The elements of an emerging pattern are usually evident after only about $30 \%$ shortening. However, in natural and experimental situations it may prove difficult to use preferred orientation as a close indication of the amount of strain since alternative (non-fabric producing) mechanisms such as diffusional mass transfer and grain boundary sliding may contribute towards bulk strain as well. Use of preferred orientation to indicate the amount of crystal plastic strain is also complicated by the effects of inhomogeneous deformation and of dynamic recrystallization. Steady state fabrics may develop in deformation zones to reflect a balance between the reorienting effects of intra- 
crystalline slip and the effects of grain boundary sliding, dynamic recrystallization and grain growth. Steady state concentrations may also be attained because for some fabrics the crystal axes do not attain rotationally stable end-orientations, but continually gyrate.

Because fabrics develop in relation to the kinematic framework on the grain scale at any instant of time, fabrics are sensitive to the closing stages of the deformation history. The skeleton of a developing pattern of preferred orientation will always be modified to reflect the influence of the closing stages of deformation. Thus pattern elements need not reflect the orientation of the kinematic framework for the whole of the deformation history, but only the last stages. In certain circumstances, for example, if dynamic recrystallization provides a continuous source of new orientations, there will be a rapid loss of information contained in the crystallographic fabric concerning earlier stages of the deformation history especially in respect to symmetry and the orientations of the instantaneous stretching axes.

Acknowledgements-The authors have benefited from discussions with numerous colleagues, in particular Mervyn Paterson, John Platt, Ernie Rutter, Jan Tullis and Paul Williams. We are indebted to Jan Tullis for a thorough review. The work commenced at the Research School of Earth Sciences, Australian National University, Canberra, Australia where concepts and programs were developed. The paper was written at the Geological Institute, University of Leiden, The Netherlands. Staff of the computer facilities at both institutions are thanked for cooperation and assistance.

\section{REFERENCES}

Baëta, R. D. \& Ashbee, K. H. G. 1969a. Slip systems in quartz: I. Experiments. Am. Miner. 54, 1551-1573.

Baêta, R. D. \& Ashbee, K. H. G. 1969b. Slip systems in quartz: II. Interpretation. Am. Miner. 54, 1574-1582.

Baěta, R. D. \& Ashbee, K. H. G. 1970. Mechanical deformation of quartz: I. Constant strain rate compression experiments. Phil. Mag. 22, 601-624.

Bell, T. H. \& Etheridge, M. A. 1976. The deformation and recrystallization of quartz in a mylonite zone, central Australia. Tectonophysics 32, 235-267.

Carreras, J., Estrada, A. \& White, S. 1977. The effects of folding on the c-axis fabrics of a quartz mylonite. Tectonophysics 39, 3-24.

Christie, J. M. 1963. The Moine Thrust zone in the Assynt region, northwest Scotland. Univ. Calif. Publs geol. Sci. 40, 345-440.

Green, H. W., Griggs, D. T. \& Christie, J. M. 1970. Syntectonic and annealing recrystallization of fine grained quartz aggregates. In: Experimental and Natural Rock Deformation (edited by Paulitsch, P.) Springer, Berlin, 272-335.

Hara, I., Takeda, K. \& Kimura, T. 1973. Preferred lattice orientation of quartz in shear deformation. J. Sci. Hiroshima Univ. Series C, 7, $1-11$.

Lister, G. S. 1979. Fabric transitions in plastically deformed quartzites: competition between basal, prism and rhomb systems. Bull. Minéral. 102, 232-41.

Lister, G. S. \& Paterson, M. S. 1979. The simulation of fabric development during plastic deformation and its application to quartzite: fabric transitions. J. Struct. Geol. 1, 99-115.

Lister, G. S., Paterson, M. S. \& Hobbs, B. E. 1978. The simulation of fabric development in plastic deformation and its application to quartzite: the model. Tectonophysics, 45, 107-158.

Lister, G. S. \& Price, G. P. 1978. Fabric development in a quartz-feldspar mylonite. Tectonophysics 49, 37-78.

Morrison-Smith, D., Paterson, M. S. \& Hobbs, B. E. 1976. An electron microscope study of plastic deformation in single crystals of synthetic quartz. Tectonophysics 33, 43-79.

Nicolas, A. \& Poirier, J. P., 1976. Crystalline Plasticity and Solid State Flow in Metamorphic Rocks. Wiley, New York.

Paterson, M. S. 1969. The ductility of rocks. In: Physics of Strength and Plasticity, The Orowan 65th Anniversary Volume (edited by Argon A. S.) The M.I.T. Press, 377-392.

Paterson, M. S. \& Weiss, L. E. 1961. Symmetry concepts in the structural analysis of deformed rocks. Bull. geol. Soc. Am. 72, 841-882.

Riekels, L. M. \& Baker, D. W. 1977. The origin of the double maximum pattern of optic axes in quartzite mylonite. J. Geol. 85, 1-16.

Tullis, J. 1977. Preferred orientation of quartz produced by slip during plane strain. Tectonophysics 39, 87-102.

Tullis, J., Christie, J. M. \& Griggs, D. T. 1973. Microstructures and preferred orientations of experimentally deformed quartzites. Bull. geol. Soc. Am. 84, 297-314.

Tullis, J. \& Tullis, T. 1972. Preferred orientation of quartz produced by mechanical Dauphine twinning: thermodynamics and axial experiments. In: Flow and Fracture of Rocks, The Griggs Volume, American Geophysical Union, 16, 67-82.

Twiss, R. J. 1976. Some planar deformation features, slip systems and submicroscopic features in synthetic quartz. J. Geol., 84, 701-724.

Weiss, L. E. 1954. A study of tectonic style-structural investigation of a marble quartzite complex. Univ. Calif. Publs geol. Sci. 30 (1), 1-102. 